\title{
Influence of Uridine Diphosphate Glucuronosyltransferase Family 1 Member A1 and Solute Carrier Organic Anion Transporter Family 1 Member B1 Polymorphisms and Efavirenz on Bilirubin Disposition in Healthy Volunteers ${ }^{\circledR}$
}

\author{
(1) Kimberly S. Collins, Ingrid F. Metzger, Brandon T. Gufford, Jessica B. Lu, Elizabeth B. Medeiros, \\ Victoria M. Pratt, Todd C. Skaar, and Zeruesenay Desta
}

Department of Medicine, Division of Clinical Pharmacology (K.S.C., I.F.M., B.T.G., J.L., T.C.S., Z.D.), and Department of Medical and Molecular Genetics (E.B.M., V.M.P.), Indiana University School of Medicine, Indianapolis, Indiana

Received August 23, 2019; accepted December 20, 2019

\begin{abstract}
Chronic administration of efavirenz is associated with decreased serum bilirubin levels, probably through induction of UGT1A1. We assessed the impact of efavirenz monotherapy and UGT1A1 phenotypes on total, conjugated, and unconjugated serum bilirubin levels in healthy volunteers. Healthy volunteers were enrolled into a clinical study designed to address efavirenz pharmacokinetics, drug interactions, and pharmacogenetics. Volunteers received multiple oral doses (600 $\mathrm{mg} /$ day for 17 days) of efavirenz. Serum bilirubin levels were obtained at study entry and 1 week after completion of the study. DNA genotyping was performed for UGT1A1 [*80 (C>T), *6 (G>A), *28 $\left(\mathrm{TA}_{7}\right),{ }^{*} 36\left(\mathrm{TA}_{5}\right)$, and *37 $\left.\left(\mathrm{TA}_{8}\right)\right]$ and for SLC01B1 [ ${ }^{* 5}(521 T>C)$ and ${ }^{*} 1 b(388 A>G]$ variants. Diplotype predicted phenotypes were classified as normal, intermediate, and slow metabolizers. Compared with bilirubin levels at screening, treatment with efavirenz significantly reduced total, conjugated, and unconjugated bilirubin. After stratification by UGT1A1 phenotypes, there was a significant decrease in total bilirubin among all phenotypes, conjugated bilirubin among intermediate metabolizers,
\end{abstract}

and unconjugated bilirubin among normal and intermediate metabolizers. The data also show that UGT1A1 genotype predicts serum bilirubin levels at baseline, but this relationship is lost after efavirenz treatment. SLCO1B1 genotypes did not predict bilirubin levels at baseline or after efavirenz treatment. Our data suggest that efavirenz may alter bilirubin disposition mainly through induction of UGT1A1 metabolism and efflux through multidrug resistance-associated protein 2.

\section{SIGNIFICANCE STATEMENT}

Efavirenz likely alters the pharmacokinetics of coadministered drugs, potentially causing lack of efficacy or increased adverse effects, as well as the disposition of endogenous compounds relevant in homeostasis through upregulation of UGT1A1 and multidrug resistance-associated protein 2. Measurement of unconjugated and conjugated bilirubin during new drug development may provide mechanistic understanding regarding enzyme and transporters modulated by the new drug.

\section{Introduction}

The human immunodeficiency virus (HIV) type 1 infection, and the AIDS associated with it, remains a major public health problem, particularly in low-income countries. The introduction of highly active antiretroviral therapy in 1996 was a landmark treatment change that considerably improved the prognosis of patients infected with HIV by substantially reducing morbidity and mortality from HIV/AIDS and by reducing incidence of HIV infection (Palella et al., 1998). Until recently, highly active antiretroviral therapy regimens containing the

This study was supported by funding from National Institutes of Health National Institute of General Medical Sciences [Grant F31-GM119401] (Collins), Clinical pharmacology Training [Grants T32-GM008425 (Gufford), R01-GM078501 (Desta), and R01-GM088076 (Skaar)], and Indiana University School of Medicine Strategic Research Initiative (Pratt/Medeiros) and the IGNITE project grant (Pratt). https://doi.org/10.1124/dmd.119.089052.

SThis article has supplemental material available at dmd.aspetjournals.org. nonnucleoside HIV-1 reverse transcriptase inhibitor efavirenz have dominated the HIV/AIDS therapy as the preferred first line in treatmentnaïve patients worldwide (Staszewski et al., 1999; Gulick et al., 2004). Efavirenz-based regimens have moved to second-line drugs in 2015 in the developed world because of the development of safe and effective antiretroviral drugs. However, efavirenz-based therapy is still widely used in many low-income countries with HIV epidemic. In addition, many patients who have been on stable efavirenz-based therapy continue this treatment (Vitoria et al., 2018).

Efavirenz shows multiple interactions with drug-metabolizing enzymes and possibly transporters (Sánchez-Martín et al., 2016; Moltó et al., 2017). As a result, efavirenz alters the pharmacokinetics of numerous drugs. These interactions can lead to reduced or lack of efficacy or increased adverse reactions. Previously, we have shown that efavirenz monotherapy affects total bilirubin disposition in healthy volunteers (Metzger et al., 2014). This effect was highly variable among subjects. The processes involved in the hepatic disposition of bilirubin

ABBREVIATIONS: CPIC, Clinical Pharmacogenetics Implementation Consortium; HIV, human immunodeficiency virus; MRP, multidrug resistance-associated protein; OATP1B, organic anion transporting polypeptide 1B; PCR, polymerase chain reactions; SLCO1B1, [*5 (521T $>$ C); SNP, single-nucleotide polymorphism; UGT1A1, uridine diphosphate glucuronosyltransferase family 1 member A1. 
are depicted in Fig. 1. Unconjugated bilirubin enters into hepatocytes from blood in part via sinusoidal membrane-bound organic anion transporting polypeptides OATP1B1 and OATP1B3 (OATP1B1/3) (Keppler, 2014) and subsequently undergoes conjugation with glucuronic acid by uridine diphosphate glucuronosyltransferase 1A1 (UGT1A1) to form bilirubin mono- and diglucuronides (Crawford et al., 1992). The glucuronides formed are either secreted into the bile mainly by multidrug resistance-associated protein 2 (MRP2) located at the canalicular membrane of hepatocytes (Jedlitschky et al., 1997) or efflux transported into the blood by MRP3 located in the hepatocyte sinusoidal membrane, and from the blood, they are reuptaken back to hepatocytes via OATP1B1/3 (van de Steeg et al., 2012; Keppler 2014). In humans, impaired bilirubin conjugation or transport leads to various degrees of hyperbilirubinemia and is observed in several inherited disorders, such as Crigler-Najjar syndrome type I and type II and Gilbert syndrome (impaired or absence of bilirubin conjugation resulting in unconjugated hyperbilirubinemia) (Bosma et al., 1995; Kadakol et al., 2000) as well as Rotor-syndrome [absence of OATP1B1/3 characterized by predominantly conjugated, and to a lesser extent unconjugated, hyperbilirubinemia because of deficient uptake transport of bilirubin, as seen in van de Steeg et al. (2012)], whereas conjugated hyperbilirubinemia because of deficient MRP2-mediated efflux transport of bilirubin glucuronides is seen in Dublin-Johnson syndrome (Sticova and Jirsa, 2013).

Because the UGT1A1 gene is a highly polymorphic enzyme, there is wide variability in enzyme activity that is associated with drug response and toxicity as well as altered bilirubin conjugation (Ah et al., 2008; Chiddarwar et al., 2017). The UGT1A1*28 ( $\left.\mathrm{TA}_{7}\right)$, a dinucleotide repeat polymorphism in the TATA sequence of the promoter region of $U G T 1 A 1$, is the most common allele associated with reduced enzyme expression and activity as well as increased serum unconjugated hyperbilirubinemia. The $U G T 1 A 1 * 28$ and other UGT1A1 missense variants have been implicated in Gilbert syndrome, an autosomal recessive unconjugated hyperbilirubinemia (Bosma et al., 1995). The Clinical Pharmacogenetics Implementation Consortium (CPIC) has published guidelines providing recommendations for the use of atazanavir, a protease inhibitor to treat HIV-1, based on UGTIAl genotypes (Gammal et al., 2016). This guideline indicates that individuals that carry two decreased function UGT1A1 alleles are at high risk for jaundice and nonadherence and recommends physicians consider an alternative drug. However, UGT1A1*28 is a TA repeat, and routine genotyping using conventional methods is challenging; however, $U G T 1 A 1 * 80($ c. $-364 \mathrm{C}>\mathrm{T})$ has been shown to be in high linkage disequilibrium with $U G T 1 A 1 * 28\left(\mathrm{TA}_{7}\right)$ and $* 37\left(\mathrm{TA}_{8}\right)$ and may serve as a tag-SNP (Gammal et al., 2016).

In this study, we hypothesize that efavirenz reduces both unconjugated and conjugated bilirubin levels primarily through induction of UGT1A1 and/or MRP2, respectively. We also explored whether: 1) variants in the $U G T 1 A 1$ and $S L C O 1 B 1$ genes influences efavirenz induction of $U G T 1 A 1$ and OATP1B1/3 and 2) $U G T 1 A 1 * 80$ could serve as easy to use surrogate for $U G T 1 A 1 * 28$ genotyping. To address these objectives, baseline (at study entry) plasma total, conjugated and unconjugated bilirubin levels were compared with those obtained 1 week after a 17-day oral treatment with $600 \mathrm{mg} /$ day efavirenz in healthy volunteers genotyped for variants in the UGT1A1 and SLCO1B1 genes.

\section{Methods}

\section{Study Population}

Eligible healthy nonsmoking 18-49-year-old male and female volunteers $(n=136)$ who were within $32 \%$ of their ideal body weight and were not taking any prescription and nonprescription medications were enrolled into two clinical trials that were designed to address efavirenz metabolism, pharmacokinetics, pharmacogenetics, and drug interactions. Study subjects were judged healthy by medical history and standard screening laboratory tests. Inclusion and exclusion criteria of the subjects have been described in detail in our previous publications (Metzger et al., 2014; Masters et al., 2016; Robarge et al., 2016). Blood samples were obtained for DNA genotyping. The studies were approved by the Indiana University School of Medicine Institutional Review Board, and each subject signed an informed consent and the Health Insurance Portability and Accountability Act documents before enrollment. The trials were conducted at the Clinical Research Center of the Indiana Clinical and Translational Sciences Institute and were registered at http://www.clinicaltrials.gov (trial identifiers NCT00668395 and NCT02401256).

\section{Study Design}

The two protocols (NCT00668395 and NCT02401256) were primarily designed to test metabolism, pharmacokinetics, pharmacogenetics, and drug interactions of efavirenz after single (600-mg) oral dose and after multiple doses (after $600 \mathrm{mg} /$ day for 17 days orally). The study designs of these trials have been described in detail elsewhere (Metzger et al., 2014; Masters et al., 2016; Robarge et al., 2016). Among others, standard laboratory results including bilirubin (total, conjugated, and unconjugated) were obtained during the screening phase (i.e., baseline, before any drug administration) and at exit (i.e., approximately 1 week after completion of $600 \mathrm{mg} /$ day oral dose of efavirenz for 17 days). In this report, we determined the influence of efavirenz chronic administration on bilirubin disposition in these trials by comparing exit plasma bilirubin concentrations (total, unconjugated, and conjugated) with those values obtained at baseline (screening).

UGT1A1 and SLCO1B1 Genotyping. Genomic DNA extracted from human blood $(n=136)$ was used for genotyping. DNA genotyping for $U G T 1 A 1 * 80$ (rs887829; $-364 \mathrm{C}>\mathrm{T}), \quad U G T 1 A 1 * 6 \quad$ (rs4148323; $211 \mathrm{G}>\mathrm{A}, \mathrm{G} 71 \mathrm{R}), \operatorname{SLCO1B1} * 5$ (rs4149056; 521T $>\mathrm{C}, \mathrm{V} 174 \mathrm{~A})$, and $S L C O 1 B 1 * 1 b(\mathrm{rs} 2306283 ; 388 \mathrm{~A}>\mathrm{G}, \mathrm{N} 130 \mathrm{D})$ was performed using TaqMan genotyping assays (Thermo Fisher Scientific, Waltham, MA) according to manufacturer's protocol on the QuantStudio 12K Flex or ViiA 7 Real-Time PCR Systems (Thermo Fisher Scientific). Genotyping for $U G T 1 A 1 * 28\left(\mathrm{TA}_{7}\right), * 36\left(\mathrm{TA}_{5}\right)$, and $* 37\left(\mathrm{TA}_{8}\right)(\mathrm{rs} 8175347)$ was performed by polymerase chain reaction (PCR) amplification and capillary electrophoresis using a protocol adapted from Huang et al. (2007). Briefly, $12 \mu$ l CloneAmp HiFi PCR Premix (Takara Bio USA, Inc., Mountain View, CA), $10.8 \mu \mathrm{l}$ water, $0.6 \mu \mathrm{l}$ forward primer 5' (6-carboxyfluorescein) CGTGACACAGTCAAACATTAACTT (0.24 $\mu \mathrm{M}$ :final concentration), and $0.6 \mu \mathrm{l}$ reverse primer 5' CAGCAGTGG CTGCCATCCAC (0.24 $\mu \mathrm{M}$ :final concentration) was PCR amplified with an initial denaturation at $98^{\circ} \mathrm{C}$ for 5 minutes, followed by 40 cycles of $98^{\circ} \mathrm{C}$ for 10 seconds, $64^{\circ} \mathrm{C}$ for 5 seconds, $72^{\circ} \mathrm{C}$ for 5 seconds, and a final extension at $72^{\circ} \mathrm{C}$ for 7 minutes. Capillary electrophoresis was performed on an Applied Biosystems 3130xL (Thermo Fisher Scientific). CPIC guidelines were used to identify UGT1A1 and SLCO1B1 phenotypes based on genotypes (Wilke et al., 2012; Gammal et al., 2016). Other variants that were genotyped in this population were described elsewhere (Robarge et al., 2016; Burgess et al., 2018).

\section{Data Analysis}

Total, conjugated, and unconjugated at screening versus exit bilirubin levels were compared using a Wilcoxon matched-pairs signed rank test for nonstratified and stratified UGT1A1 phenotypic groups. Total, conjugated, and unconjugated bilirubin level changes were compared across predicted UGT1A1 phenotypes using Kruskal-Wallis followed 


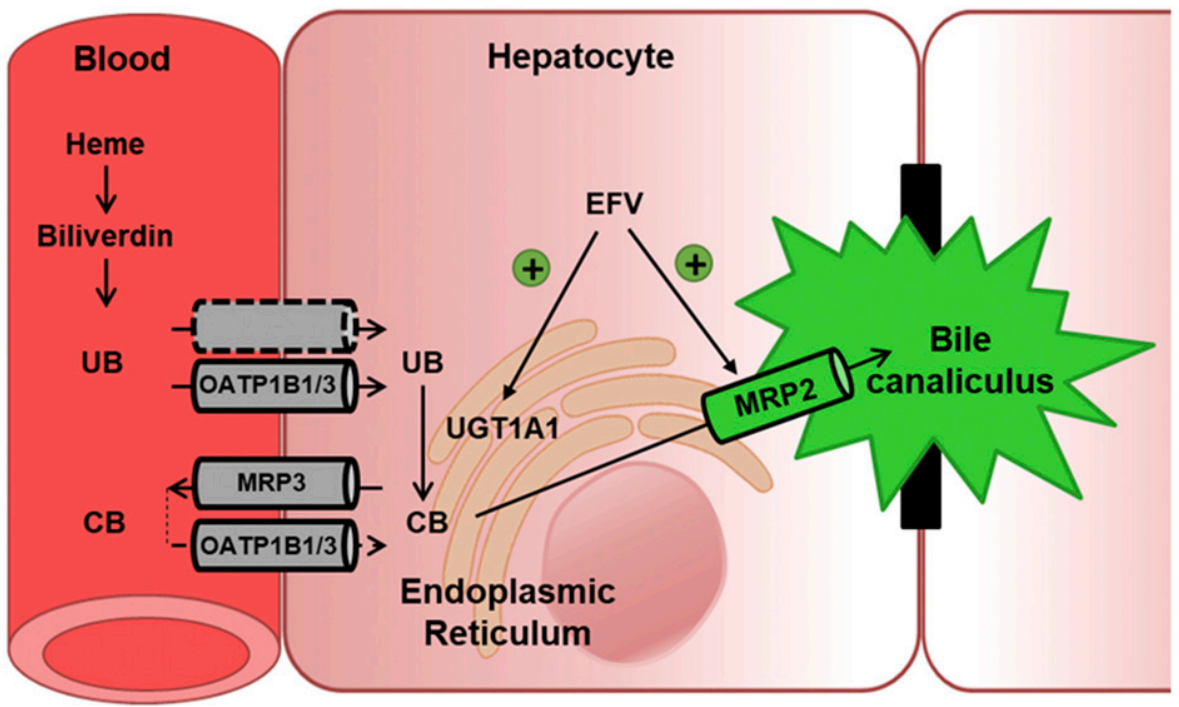

Fig. 1. Bilirubin conjugation and transport in the liver. Unconjugated bilirubin (UB), a product of heme catabolism, is uptake transported into hepatocytes in part by OATP1B1/3 and unknown transported as well as by diffusion. In hepatocytes, UB undergo UGT1A1mediated glucuronidation to mono- and biglucuronides. Conjugated bilirubin $(\mathrm{CB})$ is then efflux transported to the bile by MRP2 and a substantial fraction efflux transported to the blood by MRP3, from where they are then subsequently reuptaken effectively back to the hepatocytes via OATP1B1/3. Efavirenz-mediated upregulation of UGT1A1 metabolism and efflux transport by MRP2 are shown by plus sign.

by Dunn's post hoc test. A $P<0.05$ was considered statistically significant. All analyses were performed using Graphpad Prism 5 (La Jolla, CA). Haplotype analysis and linkage disequilibrium was calculated using SHEsis (Shi and He, 2005; Li et al., 2009).

\section{Results}

\section{Demographics}

Demographic characteristics of the study participants are listed in Table 1 . The study included 136 volunteers, mostly male (59\%) and selfidentified white $(65 \%)$. Both screening and exit total bilirubin concentrations were available for analysis in 133 study participants, whereas conjugated and unconjugated bilirubin levels were obtained in 65 and 67 subjects, respectively. The remaining subjects were missing either a screening or exit bilirubin measurement and are excluded from paired analysis only.

UGT1A1 and SLCO1B1 Genotypes and Genotype-Predicted Phenotype Frequencies. There were 136 volunteers genotyped for UGT1A1 rs887829, rs8175347, and rs4148323 as well as SLCO1B1 rs4149056 and rs2306283 (Table 2). One goal of this study was to test whether $U G T 1 A 1 * 80$ could be used as a tag-SNP for $U G T 1 A 1 * 28$. $U G T 1 A 1 * 80$ was observed at a minor allele frequency of $32.4 \%$, $U G T 1 A 1 * 28$ and $U G T 1 A 1 * 37$ were observed at a frequency of $33.5 \%$, and $U G T 1 A 1 * 6$ was at a frequency of $0.37 \%$. The $U G T 1 A 1 * 80$ is in high linkage disequilibrium in our study population with $U G T 1 A 1 * 28$ and $U G T 1 A 1 * 37\left(r^{2}=0.96\right)$; however, this was not perfect, and $2 \%$ of

TABLE 1

Demographics of study population

Continuous variables reported as average (range). Categorical variables reported as the count (percentage)

\begin{tabular}{lc}
\hline & $N=136$ \\
\hline Age (range) & $27(18-50)$ \\
Weight (range) & $73.3(52.3-109)$ \\
BMI (range) & $24.4(17.8-32.2)$ \\
Male, $n(\%)$ & $80(59)$ \\
Ethnicity & \\
White, $n(\%)$ & $89(65.4)$ \\
Black, $n(\%)$ & $23(17)$ \\
Asian, $n(\%)$ & $12(8.8)$ \\
Other, $n(\%)$ & $12(8.8)$ \\
\hline
\end{tabular}

subjects would be misclassified as $U G T 1 A 1 * 28$ when $* 80$ is used as a tag-SNP. Genotype-predicted phenotypes for UGT1A1 in the healthy volunteers were as follows: $44 \%, 47 \%$, and $9 \%$, genotypic normal, intermediate, and slow metabolizers, respectively (Table 3 ). $S L C O 1 B 1 * 5$ and $S L C O 1 B 1 * 1 b$ genotype count and frequencies are described in Table 2 and genotype-predicted phenotypes in Table 3.

\section{Efavirenz Treatment Leads to Reduced Bilirubin Levels}

Compared with screening values, exit bilirubin levels were significantly decreased after efavirenz treatment of total bilirubin $(33 \%, P<$ $0.0001)$, conjugated bilirubin $(31 \%, P=0.0004)$, and unconjugated bilirubin $(36 \%, P<0.0001)$ (Fig. 2, respectively).

UGT1A1 Polymorphisms Are Associated with Interindividual Variability in Total and Unconjugated Bilirubin Levels. Total bilirubin levels at the start of the study (screening) vary depending on UGT1A1 metabolizer status. There were $81 \%$ and $63 \%$ higher total bilirubin levels in slow metabolizers, respectively, compared with normal $(P<0.01)$ and intermediate $(P<0.05)$ metabolizers (Fig. 3A). However, this genotype-dependent effect is lost after chronic efavirenz dosing (exit), although the slow metabolizers have $50 \%$ and $46 \%$ higher total bilirubin levels on average compared with the normal

TABLE 2

UGT1A1 and SLCO1B1 genotype counts and frequencies in population

\begin{tabular}{lccrc}
\hline & & Genotype & Count & Frequency \\
\hline UGT1A1 & rs887829 & $* 1 / * 1$ & 61 & 0.449 \\
& & $* 1 / * 80$ & 62 & 0.456 \\
& & $* 80 / * 80$ & 13 & 0.096 \\
& rs8175347 & $* 1 / * 1$ & 60 & 0.441 \\
& & $* 1 / * 28$ & 54 & 0.397 \\
& & $* 1 / * 36$ & 1 & 0.007 \\
& & $* 1 / * 37$ & 5 & 0.037 \\
& & $* 28 / * 28$ & 12 & 0.088 \\
& & $* 28 / * 36$ & 4 & 0.029 \\
& & $* 1 / * 1$ & 135 & 0.993 \\
& & $* 1 / * 6$ & 1 & 0.007 \\
& & $* 1 / * 1$ & 111 & 0.810 \\
& & $* 1 / * 5$ & 17 & 0.124 \\
& & $* 5 / * 5$ & 8 & 0.058 \\
& & $* 1 / * 1$ & 38 & 0.279 \\
& & $* 1 / * 1 \mathrm{~b}$ & 54 & 0.397 \\
& & $* 1 \mathrm{~b} / * 1 \mathrm{~b}$ & 44 & 0.324 \\
\hline
\end{tabular}


TABLE 3

CPIC classification of UGT1A1 and SLCO1B1 phenotypes based on genotypes

\begin{tabular}{llcrc}
\hline & & \multicolumn{1}{c}{ Genotypes } & Count & Frequency \\
\hline \multirow{2}{*}{ UGT1A1 } & Normal & $* 1 / * 1, * 1 / * 36, * 36 / * 36$ & 60 & 0.441 \\
& Intermediate & $* 1 / * 28, * 1 / * 37$ & 64 & 0.471 \\
& & $* 36 / * 28, * 1 / * 6$ & & \\
\multirow{5}{*}{ SLCO1B1 1 Slow } & $* 28 / * 28, * 37 / * 37$ & 12 & 0.088 \\
& Normal & $* 1 / * 1, * 1 / * 1 \mathrm{~b}, * 1 \mathrm{~b} / * 1 \mathrm{~b}$ & 111 & 0.816 \\
& Intermediate & $* 1 / * 5, * 1 / * 15$ & 17 & 0.125 \\
& Slow & $* 5 / * 5, * 15 / * 15$ & 8 & 0.059 \\
\hline
\end{tabular}

and intermediate metabolizer status, respectively (Fig. 3B). No statistically significant changes were observed in conjugated bilirubin levels across UGT1A1 phenotypes at screening and exit (Fig. 3, C and D). Unconjugated bilirubin levels were $111 \%(P<0.001)$ and $73 \%(P<0.01)$ higher in the slow metabolizer group compared with the normal and intermediate metabolizers, respectively (Fig. 3E). These differences were not statistically significant after chronic efavirenz treatment (Fig. 3F).

The effect of efavirenz on total bilirubin levels stratified by each UGT1A1 genotype is illustrated in Fig. 4A. Total bilirubin levels significantly decreased after completion of efavirenz treatment in all genotypic groups, normal $(29 \%, P<0.0001)$, intermediate $(35 \%$, $P<0.0001$ ), and slow (41\%, $P=0.0078)$ metabolizers (Fig. 4A). Conjugated bilirubin levels were significantly reduced only among intermediate metabolizers (44\%, $P=0.004$ ) (Fig. 4B), and unconjugated bilirubin levels were decreased among normal $(26 \%, P=0.007)$ and intermediate metabolizers $(41 \%, P<0.0001)$ (Fig. 4C). The slow metabolizers showed a decreasing trend (52\%) with unconjugated bilirubin levels but did not meet statistical significance, probably because of small sample size.

SLCO1B1 Polymorphisms Not Associated with Interindividual Variability in Bilirubin Levels. There were no changes observed between total, conjugated, and unconjugated bilirubin levels across genotype-predicted phenotypes at screening or after completion of study (exit) (Supplemental Fig. 1). Total bilirubin levels significantly decreased after the completion of efavirenz treatment in genotypic normal $(P<0.0001)$ and intermediate $(P<0.0001)$ metabolizers (Supplemental Fig. 2A). Conjugated bilirubin levels were significantly reduced only among normal metabolizers $(P<0.01)$, and unconjugated bilirubin levels were decreased in normal $(P<0.0001)$ and intermediate $(P<0.05)$ metabolizers (Supplemental Fig. 2, B and $C$ ). No changes were observed when stratifying UGT1A1 normal metabolizers only (Supplemental Fig. 3).

\section{Discussion}

We found that variants in the UGT1A1 gene, but not SLCO1B1 genotypes, are associated with baseline bilirubin plasma concentrations; i.e., total bilirubin was significantly higher in slow metabolizers compared with normal and intermediate metabolizers. Slow metabolizers had slightly higher total bilirubin after efavirenz treatment, but the associations with genotype-predicted phenotypes did not reach a statistically significant level. Though efavirenz significantly reduced total bilirubin concentrations in all genotypes, this effect was more pronounced in UGT1A1 genotypic slow metabolizers, as there was a $41 \%$ decrease in total bilirubin levels versus a $29 \%$ and $35 \%$ decrease among normal and intermediate metabolizers, respectively. Certain drugs (e.g., atazanavir and indinavir) have been shown to increase bilirubin levels through inhibition of UGT1A1, and this interaction has been associated with hyperbilirubinemia, jaundice, and premature discontinuation of treatment, particularly in those with UGT1A1*28 alleles (Rotger et al., 2005; Gammal et al., 2016). Our data suggests that UGT1A1 genotype-dependent differential induction of UGT1A1 activity by efavirenz may occur, although the sample size in the slow metabolizers is relatively small. If this finding is confirmed in a larger sample size, efavirenz interaction with UGT1A1 substrates may be expected to depend on UGT1Al genotypes such that the magnitude of efavirenz interaction with substrates of UGT1A1 would be greater in slow metabolizer, potentially necessitating dose adjustment when coadministered with efavirenz (e.g., in patients with Gilbert syndrome).

We found that chronic administration of efavirenz significantly reduced total, unconjugated, and conjugated (glucuronides) bilirubin by $33 \%, 36 \%$, and $31 \%$, respectively. These findings concur with our previous study showing a $30 \%$ decrease in total bilirubin levels by efavirenz (Metzger et al., 2014) and with those from other investigators in which efavirenz was shown to decrease unconjugated and conjugated bilirubin by $42 \%$ and $26 \%$, respectively, in a small number of healthy volunteers (Lee et al., 2012). Although the exact mechanisms whereby efavirenz reduces bilirubin levels remains unknown, a useful, plausible insight can be obtained from a close look at the processes responsible for the hepatic disposition of unconjugated and conjugated bilirubin (Crawford et al., 1992; Bosma et al., 1995; Jedlitschky et al., 1997; Kadakol et al., 2000; van de Steeg et al., 2012; Sticova and Jirsa, 2013; Keppler, 2014) (see also Fig. 1). Multiple transporters have been implicated in bilirubin hepatic disposition, but UGT1A1 and MRP2 appear to mediate the effect of efavirenz on unconjugated and conjugated bilirubin levels.

In vitro and/or in vivo evidence has shown efavirenz-mediated pregnane $\mathrm{X}$ receptor activation (Sharma et al., 2013) to induce UGT1A1 and MRP2 expression (Gwag et al., 2019) and efavirenz-mediated
A

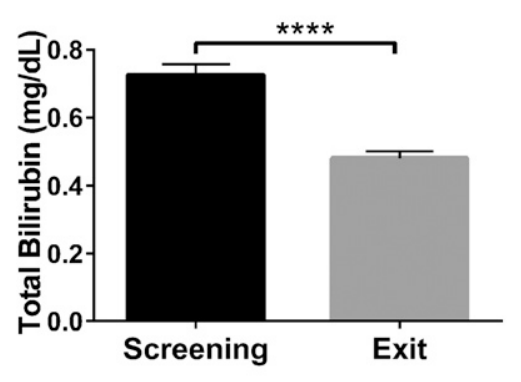

B

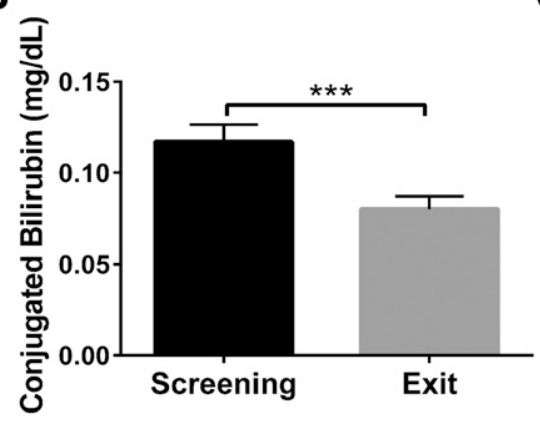

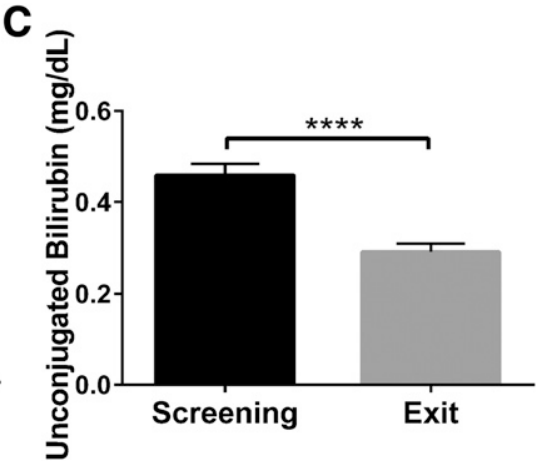

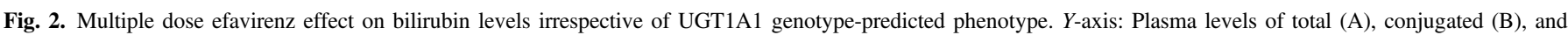

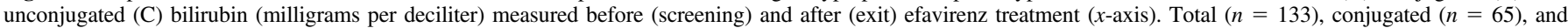
unconjugated bilirubin levels $(n=67)$. *** $P<0.001$; **** $P<0.0001$. 
A

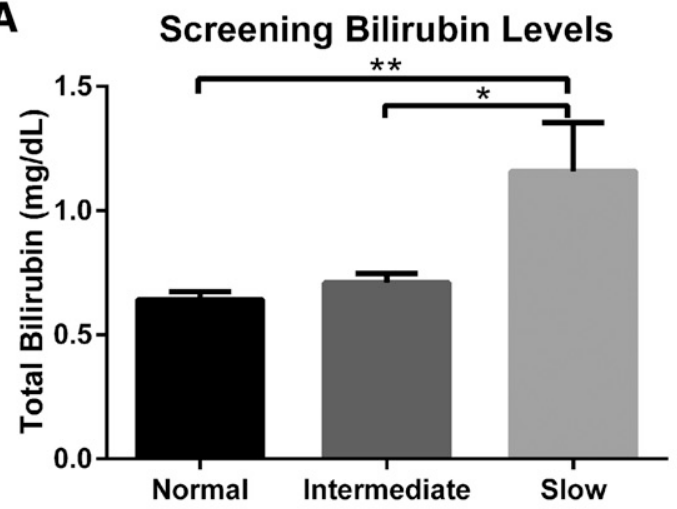

C

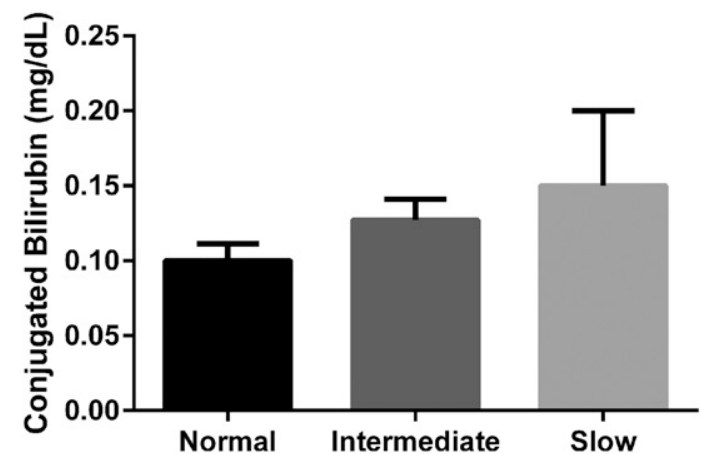

E

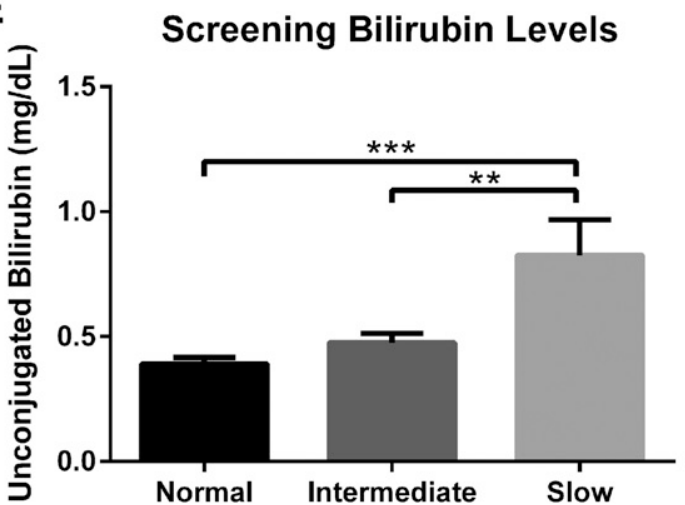

B

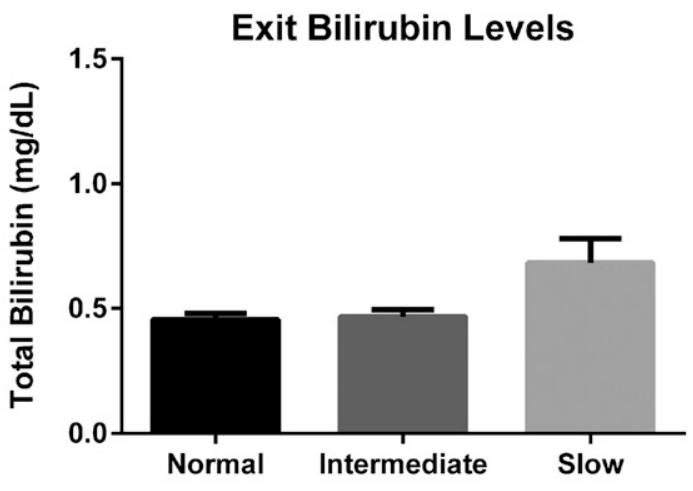

D

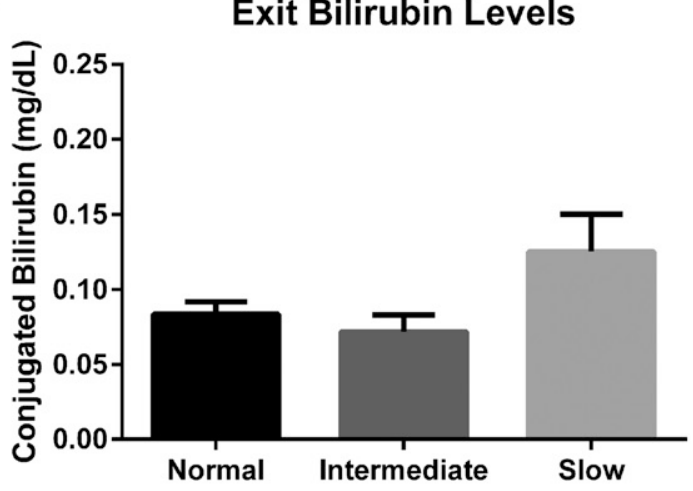

$\mathbf{F}$

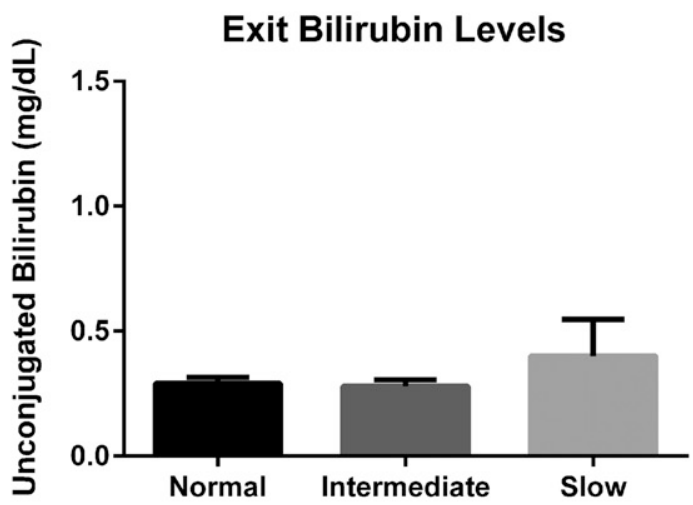

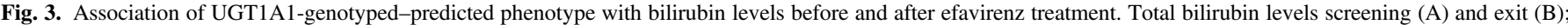

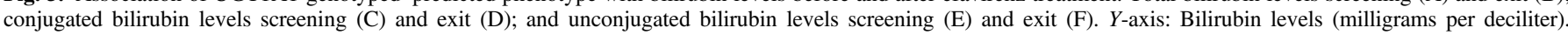

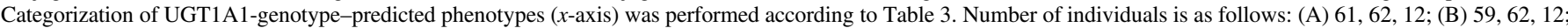
(C) $31,33,4$; (D) 30, 32, 4; (E) 32, 33, 4; and (F) 30, 33, 4. *P<0.05; ** $P<0.01$; *** $P<0.001$.

constitutively androgen receptor activation to induce UGT1Al expression (Meyer zu Schwabedissen et al., 2012). Considering that unconjugated bilirubin is a substrate of UGT1A1 (Crawford et al., 1992), and efavirenz substantially enhances the clearance of UGT1A1 substrates such as dolutegravir (Song et al., 2014), we believe that the observed decrease in unconjugated bilirubin by chronic administration of efavirenz is primarily due to induction of UGT1A1. Of note, the most potent inhibitor of UGT1A1 atazanavir results in unconjugated hyperbilirubinemia in HIV patients (Gammal et al., 2016), indicating that UGT1A1 is a rate-limiting step in its hepatic clearance. Hepatic uptake of unconjugated bilirubin from blood into hepatocytes has been shown to be mediated in part via OATP1B1/3 transporters (Keppler, 2014)
(Fig. 1), and this was confirmed in humans for whom increased unconjugated bilirubin was noted in complete absence of OATP1B1/ 3 , as is the case with Rotor syndrome (van de Steeg et al., 2012). However, associations of reduced function variants in the SLCO1B1 gene with unconjugated bilirubin level were inconsistent, which included no significant associations (our present data; $\mathrm{Hu}$ and Tomlinson, 2012; Bai et al., 2019) or significant associations (Zhang et al., 2007). Although chronic administration of efavirenz significantly reduces plasma exposure of the OATP1B1/3 and MRP2 substrate pravastatin (Gerber et al., 2005), efavirenz did not affect the exposure of a more selective OATP1B1/3 substrate pitavastatin (Malvestutto et al., 2014), further supporting that efavirenz interaction with OATP1B $1 / 3$, if 
A

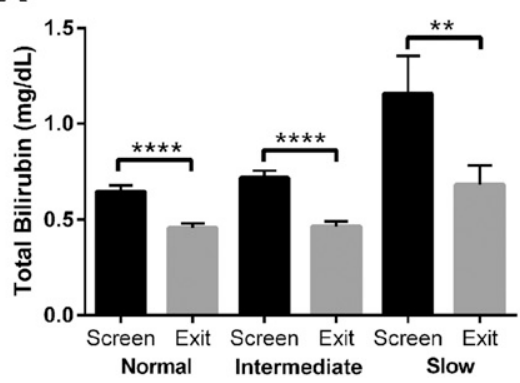

B

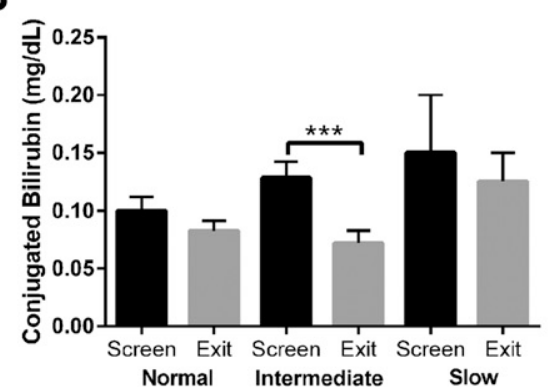

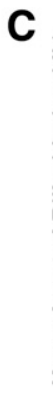

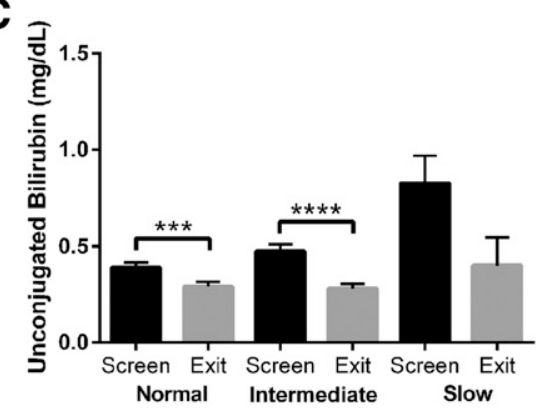

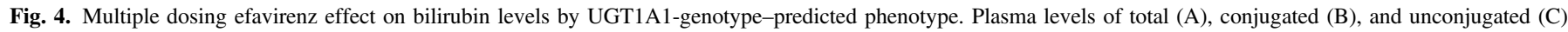

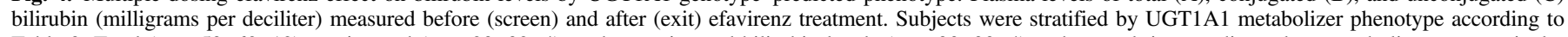

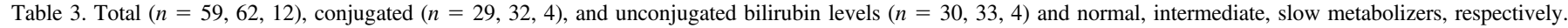
** $P<0.01$; *** $P<0.001$; **** $P<0.0001$

any, is likely marginal. Together, these data suggest that the role of OATP1B $1 / 3$ in hepatic uptake of unconjugated bilirubin is apparent only under extreme transporter functional deficiencies (van de Steeg et al., 2012) and that efavirenz and variants in the SLCO1B1 gene may not be effective modulators of unconjugated bilirubin.

Efavirenz induction of UGT1A1-mediated metabolism of unconjugated bilirubin would have been expected to increase plasma bilirubin glucuronides, but we found the opposite, i.e., efavirenz treatment decreased bilirubin conjugates. Bilirubin glucuronides are good substrates of MRP2, MRP3, and OATP1B1/3 (Fig. 1). Indeed, inherited OATP1B1/3 are associated with conjugated (and to some extent unconjugated) hyperbilirubinemia, as seen with Rotor syndrome, whereas inherited deficiency in MRP2 results in conjugated hyperbilirubinemia, as seen in human Dubin-Johnson syndrome (Keppler, 2014; van de Steeg et al., 2012). As described above, the effect of efavirenz on OATP1B1/3 is likely marginal, and, although efavirenz may show induction of mRNA of MRP3 in vitro (Weiss et al., 2009), the reuptake mechanism for bilirubin glucuronides by OATP1B1 is too efficient and rate limiting to have an impact on plasma concentrations of these metabolites. In fact, there appears to be no evidence that efavirenz may affect MRP3 clinically (Kharasch et al., 2012). Though a small contribution of MRP3 or OATP1B1/3 cannot be fully excluded, we believe that a concomitant efficient induction of MRP2-mediated efflux transport to the bile by efavirenz leads to a net reduction in conjugated bilirubin. Of note, efavirenz significantly reduces plasma exposure of pravastatin (OATP1B1/3 and MRP2 substrate) (Gerber et al., 2005), whereas no such effect is observed on the exposure of pitavastatin (a selective OATP1B1/3 substrate) (Malvestutto et al., 2014), providing further evidence that the efavirenz-pravastatin is likely due to induction of $M R P 2$.

Reduction of bilirubin levels by efavirenz may have clinical relevance. Mildly elevated serum bilirubin levels are associated with reduced risk for oxidative stress and cardiovascular disease for individuals with HIV- and non-HIV-infected patients. Elevated levels of unconjugated bilirubin observed because of variants in the UGT1A1 gene (e.g., UGT1A1*28 in Gilbert syndrome) (Burchell and Hume, 1999) or concomitant use of drugs that inhibit UGT1A1 activity (Gammal et al., 2016) have been shown to reduce cardiovascular risks or markers of cardiovascular risks (Vítek et al., 2002); Lin et al., 2006; Estrada et al., 2016). There is clinical evidence that atazanavir (a clinical inhibitor of UGT1A1) containing HIV therapy reduces cardiovascular risk when compared with efavirenz-based regimen (Estrada et al., 2016; LaFleur et al., 2017). The possibility that the substantial reduction in serum bilirubin levels during chronic treatment with efavirenz or other inducers would diminish the favorable effect of bilirubin on oxidative stress and cardiovascular outcomes cannot be ruled out and needs further study.

In summary, our data suggest that efavirenz is an effective inducer of UGT1A1 (indicated by reduction in unconjugated bilirubin) and MRP2 (indicated by significant changes in conjugated bilirubin). Efavirenz likely alters the disposition of UGT1A1 and MRP2 substrate drugs, potentially causing lack of efficacy or increased adverse effects, and endogenous compounds relevant in homeostasis. Measurement of unconjugated and conjugated bilirubin during new drug development may provide mechanistic understanding regarding enzyme and transporters modulated. Our data indicate that UGT1Al genotype, but not SLCO1B1 genotypes, predict serum bilirubin levels at baseline. Also, efavirenz treatment somewhat abrogated UGT1A1 genetic associations with bilirubin disposition. Although UGT1A1 or SLCO1B1 genotypedependent induction of bilirubin disposition was suggested, further studies are needed to confirm this trend in an adequate sample size. Lastly, even though $U G T 1 A 1 * 80$ and $* 28$ are in strong linkage disequilibrium $\left(r^{2}=0.96\right)$, few subjects would be misclassified as $* 28(2 \%)$ when $* 80$ is used as a tag-SNP. Although genotyping for $* 28$ is more accurate when feasible, genotyping for $* 80$ can be used if assays for $* 28$ are not available.

\section{Authorship Contributions}

Participated in research design: Collins, Gufford, Pratt, Skaar, Desta.

Conducted experiments: Collins, Metzger, Lu, Medeiros, Pratt.

Contributed new reagents or analytic tools: Desta.

Performed data analysis: Collins, Metzger, Gufford, Medeiros, Pratt, Desta. Wrote or contributed to the writing of the manuscript: Collins, Gufford, Pratt, Skaar, Desta.

\section{References}

Ah YM, Kim YM, Kim MJ, Choi YH, Park KH, Son IJ, and Kim SG (2008) Drug-induced hyperbilirubinemia and the clinical influencing factors. Drug Metab Rev 40:511-537.

Bai J, Luo L, Liu S, Liang C, Bai L, Chen Y, Zheng S, and Duan Z (2019) Combined effects of UGTIA1 and SLCOIBI variants on Chinese adult mild unconjugated hyperbilirubinemia. Front Genet 10:1073.

Bosma PJ, Chowdhury JR, Bakker C, Gantla S, de Boer A, Oostra BA, Lindhout D, Tytgat GN, Jansen PL, Oude Elferink RP, et al. (1995) The genetic basis of the reduced expression of bilirubin UDP-glucuronosyltransferase 1 in Gilbert's syndrome. N Engl J Med 333:1171-1175. Burchell B and Hume R (1999) Molecular genetic basis of Gilbert's syndrome. J Gastroenterol Hepatol 14:960-966.

Burgess KS, Ipe J, Swart M, Metzger IF, Lu J, Gufford BT, Thong N, Desta Z, Gaedigk R, Pearce RE, et al. (2018) Variants in the CYP2B6 3'UTR alter in vitro and in vivo CYP2B6 activity: potential role of MicroRNAs. Clin Pharmacol Ther 104:130-138.

Chiddarwar AS, D'Silva SZ, Colah RB, Ghosh K, and Mukherjee MB (2017) Genetic variations in bilirubin metabolism genes and their association with unconjugated hyperbilirubinemia in adults. Ann Hum Genet 81:11-19.

Crawford JM, Ransil BJ, Narciso JP, and Gollan JL (1992) Hepatic microsomal bilirubin UDPglucuronosyltransferase. The kinetics of bilirubin mono- and diglucuronide synthesis. J Biol Chem 267:16943-16950.

Estrada V, Monge S, Gómez-Garre MD, Sobrino P, Masiá M, Berenguer J, Portilla J, Viladés C, Martínez E, and Blanco JR; CoRIS and the HIV Biobank integrated in the Spanish AIDS 
Research Network (2016) Relationship between plasma bilirubin level and oxidative stress markers in HIV-infected patients on atazanavir- vs. efavirenz-based antiretroviral therapy. HIV Med 17:653-661.

Gammal RS, Court MH, Haidar CE, Iwuchukwu OF, Gaur AH, Alvarellos M, Guillemette C, Lennox JL, Whirl-Carrillo M, Brummel SS, et al.; Clinical Pharmacogenetics Implementation Consortium (2016) Clinical pharmacogenetics implementation consortium (CPIC) guideline for UGT1A1 and atazanavir prescribing. Clin Pharmacol Ther 99:363-369.

Gerber JG, Rosenkranz SL, Fichtenbaum CJ, Vega JM, Yang A, Alston BL, Brobst SW, Segal Y, and Aberg JA; AIDS Clinical Trials Group A5108 Team (2005) Effect of efavirenz on the pharmacokinetics of simvastatin, atorvastatin, and pravastatin: results of AIDS Clinical Trials Group 5108 Study. J Acquir Immune Defic Syndr 39:307-312.

Gulick RM, Ribaudo HJ, Shikuma CM, Lustgarten S, Squires KE, Meyer WA III, Acosta EP, Schackman BR, Pilcher CD, Murphy RL, et al.; AIDS Clinical Trials Group Study A5095 Team (2004) Triple-nucleoside regimens versus efavirenz-containing regimens for the initial treatment of HIV-1 infection. $N$ Engl J Med 350:1850-1861.

Gwag T, Meng Z, Sui Y, Helsley RN, Park SH, Wang S, Greenberg RN, and Zhou C (2019) Nonnucleoside reverse transcriptase inhibitor efavirenz activates PXR to induce hypercholesterolemia and hepatic steatosis. J Hepatol 70:930-940.

Hu M and Tomlinson B (2012) Effects of statin treatments and polymorphisms in UGT1A1 and SLCO1B1 on serum bilirubin levels in Chinese patients with hypercholesterolaemia. Atherosclerosis 223:427-432.

Huang CK, Dulau A, Su-Rick CJ, and Pan Q (2007) Validation of rapid polymerase chain reactionbased detection of all length polymorphisms in the UGT 1A1 gene promoter. Diagn Mol Patho 16:50-53.

Jedlitschky G, Leier I, Buchholz U, Hummel-Eisenbeiss J, Burchell B, and Keppler D (1997) ATPdependent transport of bilirubin glucuronides by the multidrug resistance protein MRP1 and its hepatocyte canalicular isoform MRP2. Biochem J 327:305-310.

Kadakol A, Ghosh SS, Sappal BS, Sharma G, Chowdhury JR, and Chowdhury NR (2000) Genetic lesions of bilirubin uridine-diphosphoglucuronate glucuronosyltransferase (UGT1A1) causing Crigler-Najjar and Gilbert syndromes: correlation of genotype to phenotype. Hum Mutat 16 297-306.

Keppler D (2014) The roles of MRP2, MRP3, OATP1B1, and OATP1B3 in conjugated hyperbilirubinemia. Drug Metab Dispos 42:561-565.

Kharasch ED, Whittington D, Ensign D, Hoffer C, Bedynek PS, Campbell S, Stubbert K, Crafford A, London A, and Kim T (2012) Mechanism of efavirenz influence on methadone pharmacokinetics and pharmacodynamics. Clin Pharmacol Ther 91:673-684.

LaFleur J, Bress AP, Rosenblatt L, Crook J, Sax PE, Myers J, and Ritchings C (2017) Cardiovascular outcomes among HIV-infected veterans receiving atazanavir. AIDS 31:2095-2106.

Lee LS, Pham P, and Flexner C (2012) Unexpected drug-drug interactions in human immunodeficiency virus (HIV) therapy: induction of UGT1A1 and bile efflux transporters by Efavirenz. Ann Acad Med Singapore 41:559-562.

Li Z, Zhang Z, He Z, Tang W, Li T, Zeng Z, He L, and Shi Y (2009) A partition-ligationcombination-subdivision EM algorithm for haplotype inference with multiallelic markers: update of the SHEsis (http://analysis.bio-x.cn). Cell Res 19:519-523.

Lin JP, O’Donnell CJ, Schwaiger JP, Cupples LA, Lingenhel A, Hunt SC, Yang S, and Kronenberg F (2006) Association between the UGT1A1*28 allele, bilirubin levels, and coronary heart disease in the Framingham Heart Study. Circulation 114:1476-1481.

Malvestutto CD, Ma Q, Morse GD, Underberg JA, and Aberg JA (2014) Lack of pharmacokinetic interactions between pitavastatin and efavirenz or darunavir/ritonavir. J Acquir Immune Defic Syndr 67:390-396.

Masters AR, Gufford BT, Lu JB, Metzger IF, Jones DR, and Desta Z (2016) Chiral plasma pharmacokinetics and urinary excretion of bupropion and metabolites in healthy volunteers. I Pharmacol Exp Ther 358:230-238.

Metzger IF, Quigg TC, Epstein N, Aregbe AO, Thong N, Callaghan JT, Flockhart DA, Nguyen AT, Stevens CK, Gupta SK, et al. (2014) Substantial effect of efavirenz monotherapy on bilirubin levels in healthy volunteers. Curr Ther Res Clin Exp 76:64-69.

Meyer zu Schwabedissen HE, Oswald S, Bresser C, Nassif A, Modess C, Desta Z, Ogburn ET, Marinova M, Lütjohann D, Spielhagen C, et al. (2012) Compartment-specific gene regulation of the CAR inducer efavirenz in vivo [published correction appears in Clin Pharmacol Ther (2013) 93:129]. Clin Pharmacol Ther 92:103-111.
Moltó J, Rajoli R, Back D, Valle M, Miranda C, Owen A, Clotet B, and Siccardi M (2017) Use of a physiologically based pharmacokinetic model to simulate drug-drug interactions between antineoplastic and antiretroviral drugs. J Antimicrob Chemother 72:805-811.

Palella FJ Jr, Delaney KM, Moorman AC, Loveless MO, Fuhrer J, Satten GA, Aschman DJ, and Holmberg SD; HIV Outpatient Study Investigators (1998) Declining morbidity and mortality among patients with advanced human immunodeficiency virus infection. $N$ Engl $J$ Med 338 853-860.

Robarge JD, Metzger IF, Lu J, Thong N, Skaar TC, Desta Z, and Bies RR (2016) Population pharmacokinetic modeling to estimate the contributions of genetic and nongenetic factors to efavirenz disposition. Antimicrob Agents Chemother 61.

Rotger M, Taffe P, Bleiber G, Gunthard HF, Furrer H, Vernazza P, Drechsler H, Bernasconi E, Rickenbach M, and Telenti A; Swiss HIV Cohort Study (2005) Gilbert syndrome and the development of antiretroviral therapy-associated hyperbilirubinemia. J Infect Dis 192:1381-1386.

Sánchez-Martín A, Cabrera Figueroa S, Cruz R, Porras-Hurtado L, Calvo-Boyero F, Rasool M, Domínguez-Gil Hurlé A, and Carracedo A; Tormes Team (2016) Gene-gene interactions between DRD3, MRP4 and CYP2B6 polymorphisms and its influence on the pharmacokinetic parameters of efavirenz in HIV infected patients. Drug Metab Pharmacokinet 31:349-355.

Sharma D, Lau AJ, Sherman MA, and Chang TK (2013) Agonism of human pregnane X receptor by rilpivirine and etravirine: comparison with first generation non-nucleoside reverse transcriptase inhibitors. Biochem Pharmacol 85:1700-1711.

Shi YY and He L (2005) SHEsis, a powerful software platform for analyses of linkage disequilibrium, haplotype construction, and genetic association at polymorphism loci. Cell Res $\mathbf{1 5}$ 97-98.

Song I, Borland J, Chen S, Guta P, Lou Y, Wilfret D, Wajima T, Savina P, Peppercorn A, Castellino S, et al. (2014) Effects of enzyme inducers efavirenz and tipranavir/ritonavir on the pharmacokinetics of the HIV integrase inhibitor dolutegravir. Eur J Clin Pharmacol 70: $1173-1179$.

Staszewski S, Morales-Ramirez J, Tashima KT, Rachlis A, Skiest D, Stanford J, Stryker R, Johnson P, Labriola DF, Farina D, et al. (1999) Efavirenz plus zidovudine and lamivudine, efavirenz plus indinavir, and indinavir plus zidovudine and lamivudine in the treatment of HIV-1 infection in adults. Study 006 Team. $N$ Engl J Med 341:1865-1873.

Sticova E and Jirsa M (2013) New insights in bilirubin metabolism and their clinical implications. World J Gastroenterol 19:6398-6407.

van de Steeg E, Stránecký V, Hartmannová H, Nosková L, Hřebíček M, Wagenaar E, van Esch A de Waart DR, Oude Elferink RP, Kenworthy KE, et al. (2012) Complete OATP1B1 and OATP1B3 deficiency causes human Rotor syndrome by interrupting conjugated bilirubin reuptake into the liver. J Clin Invest 122:519-528.

Vítek L, Jirsa M, Brodanová M, Kalab M, Marecek Z, Danzig V, Novotný L, and Kotal P (2002) Gilbert syndrome and ischemic heart disease: a protective effect of elevated bilirubin levels. Atherosclerosis 160 (2):449-456, doi: 10.1016/s0021-9150(01)00601-3 11849670.

Vitoria M, Hill A, Ford N, Doherty M, Clayden P, Venter F, Ripin D, Flexner C, and Domanico PL (2018) The transition to dolutegravir and other new antiretrovirals in low-income and middleincome countries: what are the issues? AIDS 32:1551-1561.

Weiss J, Herzog M, König S, Storch CH, Ketabi-Kiyanvash N, and Haefeli WE (2009) Induction of multiple drug transporters by efavirenz. J Pharmacol Sci 109:242-250.

Wilke RA, Ramsey LB, Johnson SG, Maxwell WD, McLeod HL, Voora D, Krauss RM, Roden DM, Feng Q, Cooper-Dehoff RM, et al.; Clinical Pharmacogenomics Implementation Consortium (CPIC) (2012) The clinical pharmacogenomics implementation consortium: CPIC guideline for SLCO1B1 and simvastatin-induced myopathy. Clin Pharmacol Ther 92:112-117.

Zhang W, He Y-J, Gan Z, Fan L, Li Q, Wang A, Liu Z-Q, Deng S, Huang Y-F, Xu L-Y, et al. (2007) OATP1B1 polymorphism is a major determinant of serum bilirubin level but not associated with rifampicin-mediated bilirubin elevation. Clin Exp Pharmacol Physiol 34 (12): 1240-1244, doi: 10.1111/j.1440-1681.2007.04798.x 17973861.

Address correspondence to: Dr. Zeruesenay Desta, Indiana University School of Medicine, Division of Clinical Pharmacology, 950 W Walnut St, R2 building, Rm E425, Indianapolis, IN 46202. E-mail: zdesta@iu.edu 